\title{
Radiographic features of anatomic relationship between impacted third molar and inferior alveolar canal on coronal CBCT images: risk factors for nerve injury after tooth extraction
}

Dongmiao Wang' ${ }^{1}$ Tangyi Lin², Yanling Wang², Chao Sun³, Lianfeng Yang ${ }^{3}$. Hongbing Jiang ${ }^{1}$, Jie Cheng ${ }^{1}$

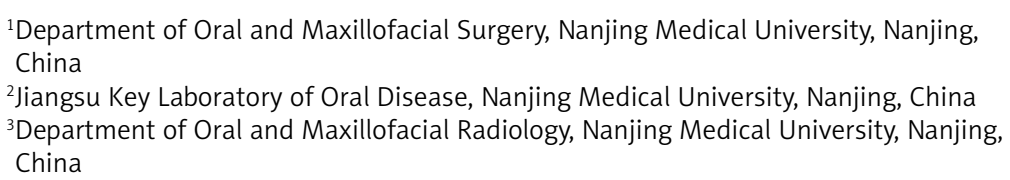

Submitted: 13 January 2016

Accepted: 27 February 2016

Arch Med Sci 2018; 14, 3: 532-540

DOI: https://doi.org/10.5114/aoms.2016.58842

Copyright (c) 2016 Termedia \& Banach

\begin{abstract}
Introduction: The present study was aimed to identify the radiographic signs between impacted third molar root and inferior alveolar canal (IAC) on cone-beam CT (CBCT) images as risk factors and prognostic predictors associated with inferior alveolar nerve (IAN) damage following tooth removal. Material and methods: A retrospective clinical study was performed involving 136 patients with 257 impacted lower third molars from January 2013 to December 2014. The neurosensory function of the lower lip and chin was subjectively evaluated and assessed by neurosensory tests before and after surgery. The preoperative $\mathrm{CBCT}$ data were retrieved and analyzed to identify the radiographic signs associated with postoperative IAN injury.

Results: The overall incidence of IAN injury in our patient cohort was $13.2 \%$. Multiple radiographic features on coronal CBCT images including contact between IAC and root, IAC position relative to root, IAC shape and cortication status were found to be significantly associated with IAN damage $(p<0.05$, $\chi^{2}$ test). Furthermore, buccolingual position, teardrop/dumbbell shape and cortication status of IAC were identified as independent prognostic predictors for IAN damage.

Conclusions: Our findings indicate that radiographic signs including direct contact between IAC and root, buccal/lingual IAC position relative to root, teardrop/dumbbell shape and cortication absence of the IAC on presurgical CBCT images are associated with high risk of IAN injury and postoperative neurosensory disturbance.
\end{abstract}

Key words: cone-beam computed tomography, inferior alveolar nerve injury, mandibular impacted molar, radiographic analysis.

\section{Introduction}

Surgical removal of the impacted mandibular third molars is one of the most frequent procedures performed by dentists and oral surgeons. Similar to other procedures, it has various types of postoperative complications including local edema, limited mouth opening, and pain [1]. However, temporary or permanent damage of the inferior alve-

\author{
Corresponding author: \\ Prof. Jie Cheng \\ Department of Oral \\ and Maxillofacial Surgery \\ Nanjing Medical University \\ 136, Hanzhong Road \\ Nanjing 210029, China \\ Phone/fax: \\ +86812585031976 \\ E-mail: leonardo_cheng@163. \\ com; omfs_njmu@163.com
}


olar nerve (IAN) is regarded as the most serious complication, with overall incidence ranging from approximately $0.4 \%$ to $13.4 \%$ [2-4]. The affected patients suffer from paresthesia or numbness of the lower lip and chin, leading to morbidity and psychological distress $[3,5]$. Therefore, it is critical to accurately evaluate the risk of IAN damage and communicate with patients about this unpleasant complication before tooth extraction.

Considering the importance of IAN impairment at third molar surgery, identifying the risk factors and preventing its occurrence are hot subjects of intensive investigations $[6,7]$. Previous studies have revealed that several clinical and anatomic parameters might be associated with IAN damage including age, gender, types of anesthesia, surgical techniques, experience of surgeons, and anatomical relationship between the inferior alveolar canal (IAC) and the third molar roots [8, 9]. Particularly, the inherent anatomical proximity between the lower third molar roots and IAN might be the principal cause and the most important predictable factor for IAN impairment after teeth extraction [7].

The importance of presurgical assessment of the anatomical relationship between the impacted third molars and IAC necessitates radiographic examinations by panoramic radiography, computed tomography (CT) or cone-beam CT (CBCT) as routine procedures before surgery in the clinic. This radiographic information is of great value for surgeons to assess the risk of IAN injury. In the panoramic images, several radiographic signs such as root darkening, loss of the IAC cortical line and defected roots have been reported to be significantly associated with increased risk of IAN damage [10, 11]. However, due to their two-dimensional nature and unequal magnification, panoramic radiographs cannot adequately meet the clinical demand to precisely observe the local anatomical relationship, thus having relatively low predictive values [12].

The advent of the CT scan enables clinicians to assess IAN risk by direct visualization from both multiple planes and 3-dimensional views [13]. Several CT radiographic features such as cortication status of IAC, IAC position and shape have been found to be associated with increased risk of IAN damage [14, 15]. However, increased cost, radiation exposure and lack of reliable diagnostic guidelines impede the routine use of CT in impacted third molar surgery. Recent introduction of CBCT in dentistry and maxillofacial surgery makes 3-dimensional radiographic examinations of the intimate relationship between roots and IAC readily available with high spatial resolution and low radiation dosage [16, 17]. The CBCT scan is becoming more and more popular in assessing IAN risk prior to lower third molar extraction and has shown advantages as compared to panoramic and CT scan [18]. However, the typical signs on CBCT images which can predict IAN damage with high reliability have not been well established yet.

The purpose of the present study was to characterize the typical radiographic features on coronal CBCT images and analyze their clinical significance as risk factors for IAN injury following impacted mandibular third molar extraction.

\section{Material and methods}

\section{Study design and patients}

This was a retrospective cohort study of patients who were referred to the Department of Oral and Maxillofacial Surgery, Affiliated Hospital of Stomatology, Nanjing Medical University, for surgical extraction of impacted mandibular third molars between January 2013 and December 2014. The research protocol was reviewed and approved by the Ethics and Research Committee of Nanjing Medical University. Written informed consent was obtained from all patients. Inclusion criteria were listed as follows: adult patients at least 18 years old with impacted lower third molars; preoperative panoramic images suggested potential risk of IAN damage during extraction; patients with preoperative CBCT images and postoperative follow-up data at least 6 months; all impacted teeth were extracted with similar approaches and atraumatic methods by the same senior surgeon with expertise and experience (Dr. Dongmiao Wang). Subjects were excluded if lesions such as a tumor or cyst around the third molar identified in CBCT images or abnormal sensory feeling in the lower lip and chin was found by patients' subjective preoperative evaluation.

\section{Morphological features and anatomic relationship between impacted teeth and mandibular canal on CBCT images}

Preoperative CBCT images of the mandible were acquired using a CBCT scanner (New Tom VG, Italy) at medium volume and high resolution. The operating parameters were $7.3 \mathrm{~mA}$ and $110 \mathrm{kV}$ with a $0.5 \mathrm{~mm}$ fixed focal spot and $18 \times 16 \mathrm{~cm}$ field of view. The local anatomy and the relationship between impacted third molars and the IAC on presurgical CBCT images were initially observed before surgery to assess the difficulties and risks of extraction.

To identify the radiographic signs as risk factors for IAN injury, we retrieved all original data of presurgical CBCT from these enrolled patients and further evaluated these data using NNT version 4.6 


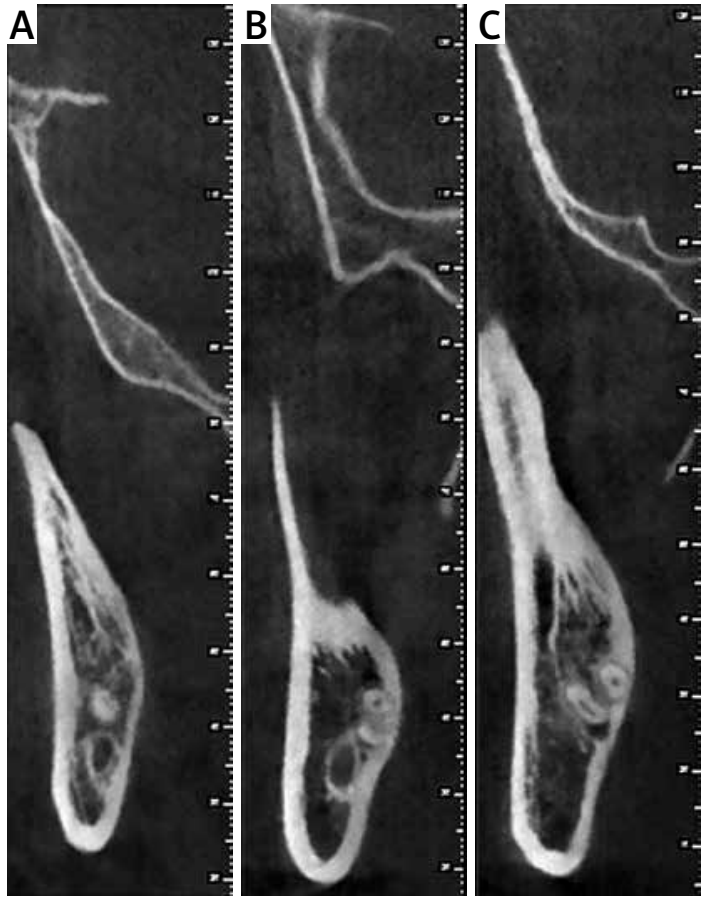

Figure 1. Radiographic images regarding direct contact between IAC and third molar roots on coronal CBCT images: $\mathbf{A}$ - no contact between IAC and root, B - direct contact between IAC and root with intact cortication of IAC, C - direct contact between $I A C$ and root with interrupted cortication of IAC software (New Tom VG, Italy). The CBCT images were evaluated from horizontal, coronal and sagittal planes independently by senior radiologists (Dr. Chao Sun and Dr. Lianfeng Yang), who were both without knowledge about patients' clinical results. When different ideas concerning the radiographic findings on CBCT images occurred, they were to discuss them with another independent observer (Dr. Tangyi Lin) and then obtain the final consensus. The anatomic and radiographic features including the relationship between the impacted third molars and the IAC, IAC position relative to the tooth root, IAC morphology as well as its cortication status were determined in the plane perpendicular to the dental arch. The anatomic relationship between the tooth root and the IAC was classified based on various factors, such as whether there was direct contact between the root and the IAC (Figure 1), buccolingual position between the IAC and the root (Figure 2), and presence or absence of IAC cortication. The morphology of IAC in the coronal plane was classified into three subtypes - round/oval, teardrop, and dumbbell - similarly as Ueda et al. reported [15] (Figure 3). Moreover, absence of IAC cortication was defined as loss of cortical lining between the root and the IAC (Figure $1 \mathrm{C}$ ).
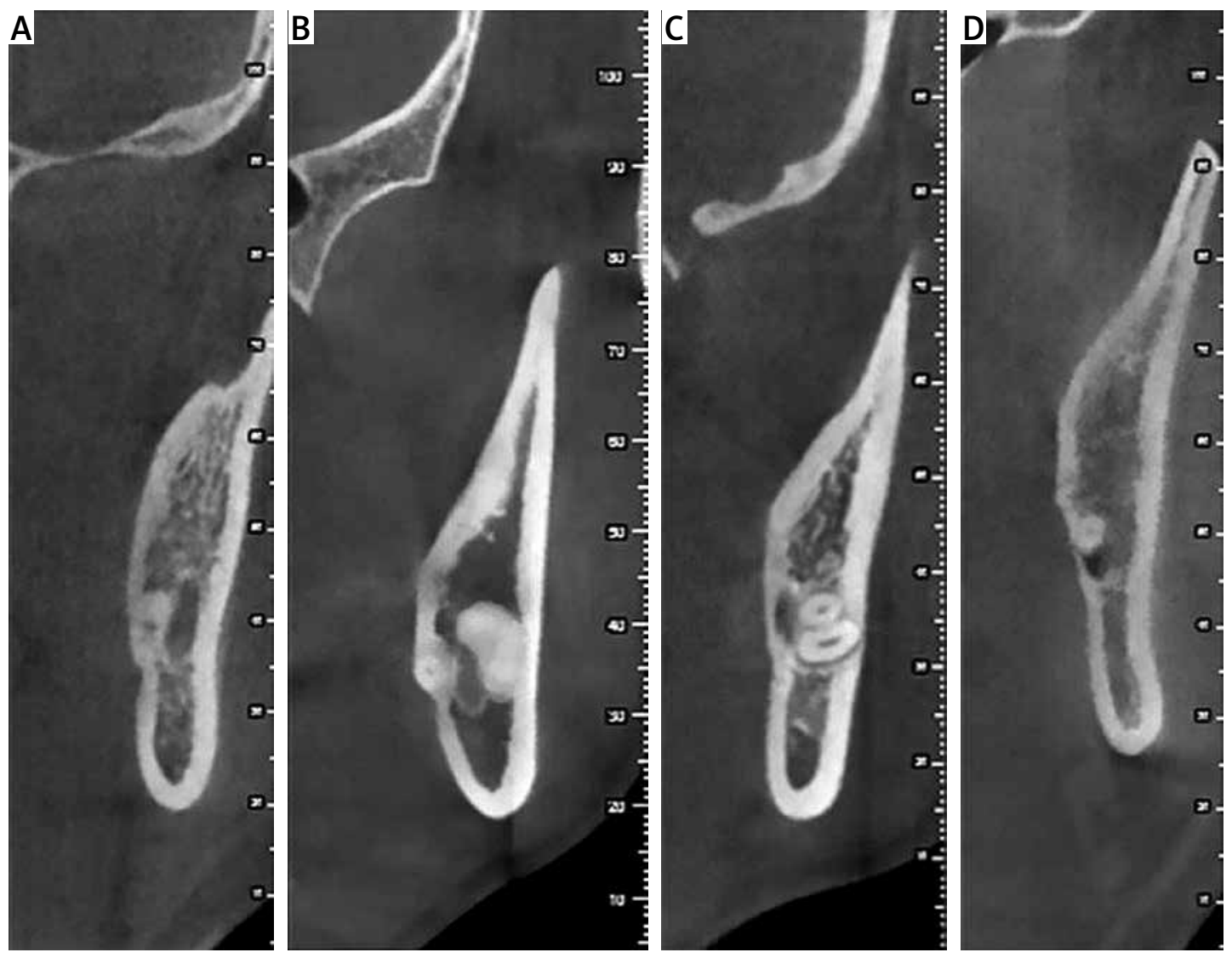

Figure 2. Radiographic images regarding IAC position relative to third molar roots on coronal CBCT images: $\mathbf{A}$ - buCcal side of IAC relative to root, $B$ - inter-radicular position of IAC, C - lingual side of IAC relative to root, D - inferior position of IAC relative to root 

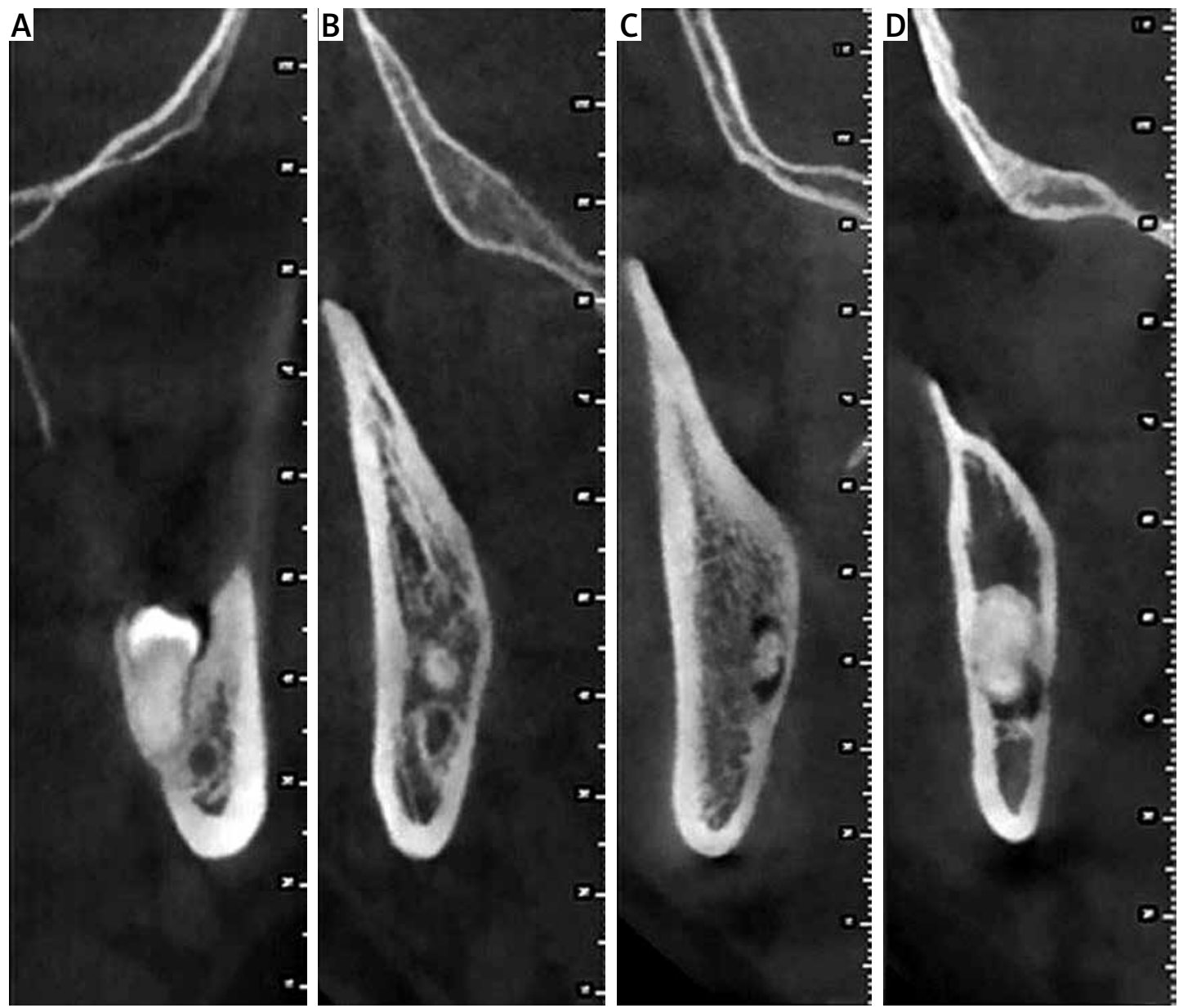

Figure 3. Radiographic images regarding IAC shape on coronal CBCT images: A - round shape of IAC, B - oval shape of IAC, C - Teardrop shape of IAC, D - Dumbbell shape of IAC

\section{Surgical approaches and techniques}

These impacted lower third molars were routinely extracted under local anesthesia. All patients were informed about potential postsurgical complications, especially IAN damage. Briefly, after local injection of saline with epinephrine (1 : 500000 dilution) to reduce bleeding, the full-thickness triangular mucoperiosteal flap was reflected carefully. The buccal and distal bone was removed to expose the impacted tooth with bur under continuous irrigation with pre-cooled sterile saline when necessary. Sometimes, the tooth crown or roots were sectioned with dental turbine handpieces. When local bony and the adjacent second molar interference was removed adequately, the tooth was extracted with a dental elevator. Then, the socket was carefully cleaned to remove debris. The surgeon (Dr. Dongmiao Wang) recorded the exposure of IAN by direct inspection of tooth sockets.

\section{Postoperative neurosensory assessment}

These patients underwent follow-up at regular intervals, at 7 days, and 1, 3, 6, and 12 months after tooth removal. The primary parameter was sensory changes in the lower lip and chin. The paresthesia or numbness in these areas reported by patients during the follow-up indicated possible IAN injury. Then, those patients with potential nerve injury were further examined by neurosensory tests including light touch, two-point discrimination and thermal stimuli by an independent examiner (Dr. Yanling Wang) who was blind to the relevant clinical data. These tests were performed and data were recorded similarly as previous reports $[19,20]$. Transient IAN injuries were defined as early paresthesia and recovery within 6 months, whereas permanent damage was defined as paresthesia or numbness incurable or unrecoverable within 6 months after surgery.

\section{Statistical analysis}

The relationships between various radiographic features and incidence of postoperative IAN damage were assessed using the $\chi^{2}$ test. Multiple epidemiological, clinical as well as radiographic parameters were analyzed using a logistic regression model to evaluate their predictive value for 
the possibility of IAN damage. Statistical analysis was performed with the Stata 9.2 software. P-value $<0.05$ (two-sided) was considered statistically significant.

\section{Results}

\section{Patients characteristics}

A total of 136 patients (68 males and $68 \mathrm{fe}-$ males) satisfying the inclusion criteria with mean age $20.2 \pm 3.90$ years old were included. These patients had 257 impacted mandibular third molars in total, with 131 on the right side and 126 on the left side. They underwent atraumatic extraction of these impacted teeth and attended regular follow-up after surgery. During the surgery, IAN exposure was observed in 62 patients. The postoperative follow-up data including subjective/ objective sensatory tests and postoperative CBCT examinations if necessary were available for evaluations of IAN status. Following extraction, $42 \mathrm{pa}-$ tients reported paresthesia in the chin and lower lip region, then received oral administration of mecobalamin for 8 weeks. Several patients recovered within 4 weeks and had normal sensory functions. Until the last follow-up at least 6 months after extraction, paresthesia or numbness was identified in 27 patients with 34 sides (15 right sides, 19 left

Table I. Associations between anatomic features and IAN injury following mandibular impacted third molar extraction

\begin{tabular}{|c|c|c|c|}
\hline \multirow{2}{*}{$\begin{array}{l}\text { Radiographic } \\
\text { findings }\end{array}$} & \multicolumn{2}{|c|}{ IAN injuries } & \multirow[t]{2}{*}{$P$-value } \\
\hline & $\begin{array}{c}\text { Yes } \\
(n=34)\end{array}$ & $\begin{array}{c}\text { No } \\
(n=223)\end{array}$ & \\
\hline \multicolumn{4}{|c|}{ Direct contact between IAC and root: } \\
\hline Yes & 29 & 141 & 0.012 \\
\hline No & 5 & 82 & \\
\hline \multicolumn{4}{|l|}{ IAC position: } \\
\hline Buccal & 14 & 26 & $<0.0001$ \\
\hline Inter-radicular & 1 & 6 & \\
\hline Lingual & 12 & 24 & \\
\hline Inferior & 7 & 167 & \\
\hline \multicolumn{4}{|l|}{ IAC shape: } \\
\hline Round/oval & 19 & 193 & 0.0001 \\
\hline Teardrop & 9 & 20 & \\
\hline Dumbbell & 6 & 10 & \\
\hline \multicolumn{4}{|c|}{ Cortication status: } \\
\hline Present & 8 & 130 & 0.0002 \\
\hline Absent & 26 & 93 & \\
\hline
\end{tabular}

sides). The incidence of permanent IAN injury was $13.2 \%$ (34/257). The postoperative CBCT images in those patients with numbness or paresthesia were used to exclude the possibility of nerve injuries resulted from alveolar bone fractures or residual displaced roots.

\section{CBCT findings of IAC and impacted tooth}

To identify the anatomic characteristics of the IAN and its relationship with impacted tooth roots which might be responsible for IAN damage, all CBCT data were retrieved and analyzed. Detailed information regarding the anatomic analyses of IAC and impacted tooth roots is presented in Table I. On the CBCT images, direct contact between the mandibular third molar and mandibular canal was observed in 130 (50.58\%) teeth. The position analyses identified 40 IACs at the buccal side of the tooth, 36 IACs at the lingual side of the tooth, 174 IACs inferior to the tooth and 7 IACs in the inter-radicular position. The most common cross-sectional shape of the IAC at the section closest to the impacted teeth was round/oval (212/257, 82.5\%), followed by teardrop and dumbbell with $11.3 \%$ and $6.2 \%$, respectively. Additionally, cortication absence of IAC was observed in 114 cases.

\section{Relationship between radiographic findings on CBCT and IAN injury}

To further assess the clinical significance of these radiographic findings, we calculated the incidence of IAN injury when these patients were categorized into diverse subgroups based on presurgical CBCT findings. As shown in Table I, significant associations were identified between IAN injury and direct contact, IAC position and shape as well as cortication status $(p<0.05)$. Notably, direct contact between the IAC and IAN, teardrop or dumbbell shape of the IAC, as well as cortication absence of the IAC suggested significantly higher incidence of IAN injury $(p<0.001)$. Furthermore, as described in Table II, IAC shapes on coronal CBCT image were significantly associated with its cortication status $(p<0.0001)$, whereas the associations between IAC position and shape,

Table II. Association between IAC shape and cortication status

\begin{tabular}{|c|c|c|c|}
\hline \multirow{2}{*}{$\begin{array}{l}\text { Radiographic } \\
\text { findings }\end{array}$} & \multicolumn{2}{|c|}{ Cortication status } & \multirow[t]{2}{*}{$P$-value } \\
\hline & Present & Absent & \\
\hline IAC shape: & & & $<0.0001$ \\
\hline Round/oval & 132 & 74 & \\
\hline Teardrop & 4 & 29 & \\
\hline Dumbbell & 2 & 16 & \\
\hline
\end{tabular}


IAC position and cortication were not statistically significant (data not shown). Additionally, intraoperative IAN exposure significantly correlated with IAN injury $(p<0.001$, Table III).

\section{Multivariate regression analyses of IAN injury after impacted mandibular third molar removal}

To determine whether these radiographic signs can be exploited as risk factors for IAN injury after tooth removal, we performed multivariate analyses via a logistic regression model using parameters including the abovementioned anatomic characteristics of the IAC and its relationship with the impacted tooth root. As shown in Table IV, several anatomic signs including teardrop and dumbbell shapes of the IAC together with cortication absence were found to be significant independent factors predicting IAN injury after surgery. These anatomic signs suggested increased risk of IAN impairment with OR values more than 1.0. In addition, as expected, anatomic signs such as no contact between the IAN and tooth root predicted much lower risk of IAN damage with an OR value of 0.14 (95\% Cl: 0.05-0.38).

\section{Discussion}

Inferior alveolar nerve damage and subsequent neurosensory disturbance following impacted lower third molar extraction is a rare but serious complication, which usually leads to functional deficit and decreased quality of life [1, 3]. Preoperative risk assessment is critical for oral surgeons to avoid or prevent IAN injury during third molar removal. Previous reports have revealed that the incidence of IAN injury following impacted mandibular third molar extraction varied from $0.4 \%$ to $13.4 \%[2,3]$. The incidence of IAN damage in the present patient cohort falls in this range (13.2\%). This incidence seems a little higher as compared with some previous reports. We reason that the patients enrolled here had an impacted third molar with relatively high risk of nerve damage and more difficulties following initial assessment in panoramic images as evidenced by the fact that $66.1 \%(170 / 257)$ of teeth had direct contact between the IAC and roots. Indeed, a large proportion of patients included here was transferred to our tertiary hospital from a local hospital or dental clinic due to surgical risks and difficulties.

The topographic relationship between the impacted third molar and IAC has been well established as the most important factor associated with postoperative sensory dysfunction, thus highlighting the necessity and importance of accurate radiographic examination of local anatomic relationships. Panoramic radiography is routinely utilized to initially assess the complicat-
Table III. Association between intraoperative IAN exposure and IAN injury

\begin{tabular}{|lccc|}
\hline $\begin{array}{l}\text { Intraoperative } \\
\text { findings }\end{array}$ & \multicolumn{2}{c|}{ IAN injuries } & P-value \\
\cline { 2 - 3 } & $\begin{array}{c}\text { Yes } \\
(n=34)\end{array}$ & $\begin{array}{c}\text { No } \\
(n=223)\end{array}$ & \\
\hline $\begin{array}{l}n=0.0001 \\
\text { IAN exposure: }\end{array}$ & 24 & 38 & \\
\hline Yes & 10 & 185 \\
\hline No & & & \\
\hline
\end{tabular}

ed third molars and estimate the risk of IAN damage. Previous studies have identified multiple radiographic signs on panoramic radiographs such as root narrowing and darkening which indicate a high risk of IAN exposure or IAN injury following third molar removal [21, 22]. However, although panoramic radiography has advantages including common availability and low cost, this imaging modality involves several potential sources of misinterpretation, such as image enlargement, distortion by projection errors, blurred images, and others [12]. These limitations significantly undermine its diagnostic accuracy and largely preclude its usage as the prerequisite measurement before surgical extraction of impacted lower third molars. However, a CT or CBCT scan can provide diagnostic information in the sagittal, axial, and coronal planes without overlap of structures [23].

Table IV. Multivariate logistic regression analyses to identify risk factors to predict IAN injury after mandibular impacted third molar extraction

\begin{tabular}{|c|c|}
\hline Risk factors & OR $(95 \% \mathrm{Cl})$ \\
\hline \multicolumn{2}{|c|}{ Direct contact between IAC and root: } \\
\hline Yes & 1 \\
\hline No & $0.14(0.05-0.38)$ \\
\hline \multicolumn{2}{|l|}{ IAC position: } \\
\hline Buccal & 1 \\
\hline Inter-radicular & $1.68(0.24-1.93)$ \\
\hline Lingual & $2.04(1.29-5.02)$ \\
\hline Inferior & $0.57(0.19-1.68)$ \\
\hline \multicolumn{2}{|l|}{ IAC shape: } \\
\hline Round/oval & 1 \\
\hline Teardrop & $4.61(1.84-11.56)$ \\
\hline Dumb bell & $5.86(1.89-18.15)$ \\
\hline \multicolumn{2}{|l|}{ Cortication status: } \\
\hline Present & 1 \\
\hline Absent & $4.51(1.95-10.41)$ \\
\hline
\end{tabular}

Cl - confidence interval, $O R$ - odds ratio. 
These two image modalities might be superior to panoramic radiography to accurately reflect the local anatomy. However, Sanmarti-Garcia et al. reported that a preoperative CT scan did not decrease the risk of IAN injury from removal of lower third molars in a retrospective cohort study [24]. In addition, the diagnostic accuracy of CBCT has been reported to be superior to or not better than panoramic images in predicting IAN exposure or damage [17, 25]. Although here we did not seek to compare the accuracy and utility of $C B C T$ and panoramic radiography in predicting IAN damage, our clinical experience and other reports tend to support the superiority of $\mathrm{CBCT} / \mathrm{CT}$ in precise assessment of the intimate relationship between the IAC and the roots $[6,25,26]$.

Some reports have revealed clinical significance of $C T$ images to assess the relationship between the IAC and the third molars [15, 24]. However, due to its relatively high cost and radiation dose, it is not recommended as the first radiographic technique of choice. Notably, CBCT provides better image quality of teeth and their surrounding structures as compared with conventional CT and seems to be a more accurate imaging modality to view the IAC from different directions [18, 27]. In the present study, we retrospectively included patients with impacted mandibular third molar extraction and sought to identify radiographic signs on $\mathrm{CBCT}$ images as risk factors to predict potential IAN injury. Here we focused on the coronal plane of CBCT mainly because it clearly and comprehensively showed the whole range of the positional relationship between the impacted third molar and the IAC, and also the morphology and cortication of the IAC in the same plane. Our data revealed that direct contact between the IAC and roots, IAC buccolingual position relative to roots, IAC shape, IAC cortication and intraoperative IAN exposure were significantly associated with postoperative IAN injury. As anticipated, direct contact between the IAC and roots indicated significantly higher risk of IAN following extraction, as previous reports established. The buccal or lingual position of the IAC relative to third molar roots was another important risk factor, since our data revealed much higher incidence of IAN injury under these two anatomic conditions. These findings were also supported by recent studies which reported that patients whose molar roots intersected with the IAC particularly on the buccal side had a higher risk of IAN damage [7, 17, 28]. Trauma during surgical extraction, for example elevator-forced root movement to compress the nerve, is likely to be responsible for IAN injury. Moreover, our data revealed that patients with both teardrop and dumbbell shapes of IAC had significantly higher incidences of IAN injury, which was in line with the report of Ueda et al. [15]. They reported that the incidence of IAN damage was relatively high in patients with the dumbbell shape of IAC, whereas the round/oval shape did not suggest high risk. Consistently, a line of evidence has revealed that IAC shape is closely associated with the root, manifested as the dumbbell/teardrop-shaped IAC usually indicating direct IAC contact with the root [15].

Furthermore, we found that the absence of IAC cortication was significantly associated with high incidence of IAN, which was in agreement with previous findings demonstrating the clinical significance of cortication status of the IAC as a reliable risk indicator for IAN injury $[15,24$, 25]. Indeed, by comparing panoramic tomography and $\mathrm{CT}$ images, studies have indicated that cortical bone loss of the IAC is one of the causes of "darkness of the root", which was one of the high risk radiographic signs observed in panoramic images [16, 25]. Moreover, a cortical defect size over $3 \mathrm{~mm}$ in length was associated with increased risk for intraoperative IAN exposure during third molar surgery [29]. More importantly, our multivariate regression analyses further revealed teardrop/ dumbbell shape of IAC and cortication absence as independent prognostic factors to predict postoperative IAN injury.

There are several advantages and limitations concerning our findings. Given that Jerjes et al. identified the experience of the surgeon or surgical skills as a major factor influencing IAN status followed by tooth status and anatomic relationship [30], tooth removal in this study was managed by the same surgeon with expertise and performed in a similar and non-invasive manner, thus reducing the factitious factors complicating the analyses. This is also supported by a recent report suggesting that besides the anatomic risks, surgical techniques such as tooth sectioning can significantly reduce the incidence of nerve damage [31]. Another merit of this study is our comprehensive analysis of the anatomical relationship between the IAC and root as well as IAC morphology and status using coronal CBCT images. Most previous studies usually included one or two radiographic characteristics to analyze the risk factors for IAN damage or exposure in third molar surgery $[7,15]$. However, a few limitations of this study exist, such as the relatively small sample size as well as possible selection bias of patients. Of course, more objective and quantitative methods to accurately clarify the anatomic relationship between the lower third molars and the IAN are still needed, although Wang et al. recently proposed a cylindrical coordinate system based on CT images as a new analysis system and provided clinical practitioners with a more explicit and objective description of the local anatomy [32]. We believe that these 
identified radiographic signs on CBCT coronal images with high risk for IAN damage are valuable and instructive for dentists and oral surgeons during treatment planning and risk assessment for impacted lower third molar extraction.

In conclusion, our study identified several radiographic characteristics on coronal CBCT images including direct contact between the IAC and root, buccal/lingual IAC position relative to root, teardrop/dumbbell shape and cortication absence of IAC, which are associated with high risk of IAN injury in impacted third molar surgery. Much attention should be paid to assessing the topographic relationship between the IAC and roots as well as the IAC buccolingual position, shape and cortication status before complicated lower third molar extraction.

\section{Acknowledgments}

This work is financially supported, in whole or in part, by the National Natural Science Foundation of China (81572669), a Project Funded by the Priority Academic Program Development of Jiangsu Higher Education Institutions (2014-37), China Postdoctoral Science Foundation (2014M560436), Jiangsu Creative Training Project for Graduates in Colleges (SJZZ_0119) and Jiangsu Creative Training Project for College Students (201510312053X).

\section{Conflict of interest}

The authors declare no conflict of interest.

\section{References}

1. Susarla SM, Blaeser BF, Magalnick D. Third molar surgery and associated complications. Oral Maxillofac Surg Clin North Am 2003; 15: 177-86.

2. Cheung LK, Leung YY, Chow LK, Wong MC, Chan EK, Fok YH. Incidence of neurosensory deficits and recovery after lower third molar surgery: a prospective clinical study of 4338 cases. Int J Oral Maxillofac Surg 2010; 39: 320-6.

3. Lopes V, Mumenya R, Feinmann C, Harris M. Third molar surgery: an audit of the indications for surgery, post-operative complaints and patient satisfaction. $\mathrm{Br} J$ Oral Maxillofac Surg 1995; 33: 33-5.

4. Valmaseda-Castellon E, Berini-Aytes L, Gay-Escoda C. Inferior alveolar nerve damage after lower third molar surgical extraction: a prospective study of 1117 surgical extractions. Oral Surg Oral Med Oral Pathol Oral Radiol Endod 2001; 92: 377-83.

5. Bataineh AB. Sensory nerve impairment following mandibular third molar surgery. J Oral Maxillofac Surg 2001; 59: 1012-7.

6. Guerrero ME, Nackaerts O, Beinsberger J, et al. Inferior alveolar nerve sensory disturbance after impacted mandibular third molar evaluation using cone beam computed tomography and panoramic radiography: a pilot study. J Oral Maxillofac Surg 2012; 70: 2264-70.

7. Xu GZ, Yang C, Fan XD, et al. Anatomic relationship between impacted third mandibular molar and the man- dibular canal as the risk factor of inferior alveolar nerve injury. Br J Oral Maxillofac Surg 2013; 51: e215-9.

8. Selvi F, Dodson TB, Nattestad A, Robertson K, Tolstunov L. Factors that are associated with injury to the inferior alveolar nerve in high-risk patients after removal of third molars. Br J Oral Maxillofac Surg 2013; 51: 868-73.

9. Hasegawa T, Ri S, Shigeta T, et al. Risk factors associated with inferior alveolar nerve injury after extraction of the mandibular third molar: a comparative study of preoperative images by panoramic radiography and computed tomography. Int J Oral Maxillofac Surg 2013; 42: 843-51.

10. Peixoto LR, Gonzaga AK, Melo SL, Pontual ML, Pontual Ados A, de Melo DP. The effect of two enhancement tools on the assessment of the relationship between third molars and the inferior alveolar canal. J Craniomaxillofac Surg 2015; 43: 637-42.

11. Monaco G, Montevecchi M, Bonetti GA, Gatto MR, Checchi L. Reliability of panoramic radiography in evaluating the topographic relationship between the mandibular canal and impacted third molars. J Am Dent Assoc 2004; 135: 312-8.

12. Kamrun N, Tetsumura A, Nomura Y, et al. Visualization of the superior and inferior borders of the mandibular canal: a comparative study using digital panoramic radiographs and cross-sectional computed tomography images. Oral Surg Oral Med Oral Pathol Oral Radiol 2013; 115: 550-7.

13. Susarla SM, Dodson TB. Preoperative computed tomography imaging in the management of impacted mandibular third molars. J Oral Maxillofac Surg 2007; 65: 83-8.

14. Nakayama K, Nonoyama M, Takaki Y, et al. Assessment of the relationship between impacted mandibular third molars and inferior alveolar nerve with dental 3-dimensional computed tomography. J Oral Maxillofac Surg 2009; 67: 2587-91.

15. Ueda M, Nakamori K, Shiratori K, et al. Clinical significance of computed tomographic assessment and anatomic features of the inferior alveolar canal as risk factors for injury of the inferior alveolar nerve at third molar surgery. J Oral Maxillofac Surg 2012; 70: 514-20.

16. Jung YH, Nah KS, Cho BH. Correlation of panoramic radiographs and cone beam computed tomography in the assessment of a superimposed relationship between the mandibular canal and impacted third molars. Imaging Sci Dent 2012; 42: 121-7.

17. Ghaeminia H, Meijer GJ, Soehardi A, Borstlap WA, Mulder J, Berge SJ. Position of the impacted third molar in relation to the mandibular canal. Diagnostic accuracy of cone beam computed tomography compared with panoramic radiography. Int I Oral Maxillofac Surg 2009; 38: 964-71.

18. Hashimoto K, Arai Y, Iwai K, Araki M, Kawashima S, Terakado M. A comparison of a new limited cone beam computed tomography machine for dental use with a multidetector row helical CT machine. Oral Surg Oral Med Oral Pathol Oral Radiol Endod 2003; 95: 371-7.

19. Ylikontiola L, Kinnunen J, Oikarinen K. Comparison of different tests assessing neurosensory disturbances after bilateral sagittal split osteotomy. Int J Oral Maxillofac Surg 1998; 27: 417-21.

20. Gianni AB, D’Orto O, Biglioli F, Bozzetti A, Brusati R. Neurosensory alterations of the inferior alveolar and mental nerve after genioplasty alone or associated with sagittal osteotomy of the mandibular ramus. J Craniomaxillofac Surg 2002; 30: 295-303. 
21. Rood JP, Shehab BA. The radiological prediction of inferior alveolar nerve injury during third molar surgery. $\mathrm{Br}$ J Oral Maxillofac Surg 1990; 28: 20-5.

22. Blaeser BF, August MA, Donoff RB, Kaban LB, Dodson TB. Panoramic radiographic risk factors for inferior alveolar nerve injury after third molar extraction. J Oral Maxillofac Surg 2003; 61: 417-21.

23. Guerrero ME, Shahbazian M, Elsiena Bekkering G, Nackaerts O, Jacobs R, Horner K. The diagnostic efficacy of cone beam CT for impacted teeth and associated features: a systematic review. J Oral Rehabil 2011; 38: 208-16.

24. Sanmarti-Garcia G, Valmaseda-Castellon E, Gay-Escoda C. Does computed tomography prevent inferior alveolar nerve injuries caused by lower third molar removal? J Oral Maxillofac Surg 2012; 70: 5-11.

25. Tantanapornkul W, Okochi K, Bhakdinaronk A, Ohbayashi N, Kurabayashi T. Correlation of darkening of impacted mandibular third molar root on digital panoramic images with cone beam computed tomography findings. Dentomaxillofac Radiol 2009; 38: 11-6.

26. Flygare L, Ohman A. Preoperative imaging procedures for lower wisdom teeth removal. Clin Oral Investig 2008; 12: 291-302.

27. Ylikontiola L, Moberg K, Huumonen S, Soikkonen K, Oikarinen K. Comparison of three radiographic methods used to locate the mandibular canal in the buccolingual direction before bilateral sagittal split osteotomy. Oral Surg Oral Med Oral Pathol Oral Radiol Endod 2002; 93: 736-42.

28. Maegawa H, Sano K, Kitagawa Y, et al. Preoperative assessment of the relationship between the mandibular third molar and the mandibular canal by axial computed tomography with coronal and sagittal reconstruction. Oral Surg Oral Med Oral Pathol Oral Radiol Endod 2003; 96: 639-46.

29. Susarla SM, Sidhu HK, Avery LL, Dodson TB. Does computed tomographic assessment of inferior alveolar canal cortical integrity predict nerve exposure during third molar surgery? J Oral Maxillofac Surg 2010; 68: 1296-303.

30. Jerjes W, El-Maaytah M, Swinson B, et al. Experience versus complication rate in third molar surgery. Head Face Med 2006; 2: 14.

31. Jain N, Thomas S, Prabhu S, et al. Influence of tooth sectioning technique and various risk factors in reducing the IAN injury following surgical removal of an impacted mandibular third molar. Oral Maxillofac Surg 2016; 20: 149-56.

32. Wang WQ, Chen MY, Huang HL, Fuh LJ, Tsai MT, Hsu JT. New quantitative classification of the anatomical relationship between impacted third molars and the inferior alveolar nerve. BMC Med Imaging 2015; 15: 59. 